\title{
Prevalence of Renal Cysts in a Group of Adult Sri Lankan Cadavers
}

\author{
Kodikara I ${ }^{1}$, Nilmini KWGP ${ }^{1}$, Hasan $\mathrm{R}^{2}$, Nanayakkara BG ${ }^{1}$, Ilayperuma I ${ }^{1}$ \\ ${ }^{\prime}$ Department of Anatomy, Faculty of Medicine, University of Ruhuna, Sri Lanka. \\ ${ }^{2}$ Department of Anatomy, Faculty of Medicine, University of Kelaniya, Sri Lanka.
}

\section{Introduction}

Cysts are the most common spaceoccupying lesion of the kidney. With the advances in imaging technology and population-based studies on the prevalence of renal cysts, their clinical importance has grown. It is known that the prevalence of renal cysts correlates with advancing age.

\section{Methods}

Present study was conducted using a total of 52 kidneys (14 male and 12 female) obtained from formalin fixed cadavers from the Universities of Ruhuna and Kelaniya, Sri Lanka. Subjects with any history of renal and vascular impairments were excluded from the study. Morphological features including presence of single or multiple cysts, their numbers and the side of the kidney were recorded.

\section{Results}

The mean age of the study population was 65 years. The incidence of renal cysts was $65.38 \%$.Cysts were more common in females $(58.82 \%)$ as compared to the males $(41.18 \%)$. The majority of cysts were solitary (70.59\%) whereas 29.41\% was multiple. Most of the renal cysts were present unilaterally (88.24\%) and $11.76 \%$ was bilateral. The cysts were common in the right kidney (64.71\%) compared to the left kidney (35.29\%).

\section{Discussion and Conclusions}

This study provides an insight regarding the incidence of renal cysts in a group of adult Sri Lankan population. The prevalence rates of renal cysts should be calculated according to different age groups since any cohort comprising a greater proportion of elderly would render higher rates. Similar studies on a younger Sri Lankan cohort are recommended. 